\title{
Approaches to turbulence in high-energy-density experiments
}

\author{
R Paul Drake, Eric C Harding and Carolyn C Kuranz \\ University of Michigan, 2455 Hayward St., Ann Arbor, MI 48105, USA \\ E-mail: rpdrake@umich.edu
}

Received 6 April 2008

Accepted for publication 28 May 2008

Published 17 December 2008

Online at stacks.iop.org/PhysScr/T132/014022

\begin{abstract}
This paper provides a discussion of the properties of hydrodynamic systems at high energy density, discusses the methods of doing hydrodynamic experiments and discusses studies to date of the three primary instabilities - Richtmyer-Meshkov (RM), Rayleigh-Taylor (RT) and Kelvin-Helmholtz (KH). The first two of these have been systematically observed, but have not yet produced a system with a clear transition to turbulence. The $\mathrm{KH}$ instability remains to be systematically observed in its pure form, although some related effects such as spike tip broadening have been seen. However, the KH effects seen in some simulations of RT systems and supersonic jets have not been seen to date in experiments. We note that the time-dependent condition for turbulence of Zhou et al (2003 Phys. Plasmas 10 1883) is roughly equivalent to the Reynolds-number threshold of Dimotakis in that eddies will dissipate by turbulence in about one eddy-turnover time. We suggest that a plausible explanation of the absence of $\mathrm{KH}$ in several experimental systems may be that finite velocity gradients have quenched the instability. Finally, we argue that despite the smearing of the shear layer caused by viscous diffusion, $\mathrm{KH}$ instabilities have the potential to contribute to the generation of fluctuations at all scales, but only if the local shear layers are initially formed with a sufficiently steep velocity gradient.
\end{abstract}

PACS numbers: 52.35.Py, 52.35.Ra, 47.20.Ft, 47.27.Cn

(Some figures in this article are in colour only in the electronic version.)

\section{Introduction}

The emergence of high-energy-density physics (HEDP) as a field of study followed the development of energy sources that can concentrate energy to densities above a kilojoule per cubic millimeter on timescales rapid compared with cooling mechanisms [1, 2], and of effective diagnostics for experiments using such sources. The present definition of the HEDP regime corresponds to one-tenth this value, which is $10^{12} \mathrm{ergs} \mathrm{cm}^{-3}$, corresponding approximately to 1 megabar (1 million atmospheres) of pressure. This definition is not without problems, but will suffice for our purposes here, where our focus will be on the fluid-like behavior of dense, ionized, high-pressure plasmas. HEDP experiments typically involve shock waves having very high Mach numbers, corresponding to strongly compressible flow. These experiments are also brief in comparison with a hydrodynamic timescale $\tau_{\mathrm{h}}$, defined as the ratio of some distance scale of interest, $\delta$, to the characteristic velocity of the flow, $U$. Experiment durations vary from of the order of $\tau_{\mathrm{h}}$ to a few tens times $\tau_{\mathrm{h}}$. This raises the natural question of whether and to what extent one may observe a transition to turbulence in HEDP flows. HEDP flows are inherently unsteady, and so the approach to turbulence in these flows is directly relevant to the 'Turbulent Mixing and Beyond' conference.

Turbulence, of course, has a wide range of definitions across subfields of physics that deal with fluid-like systems, so some discussion of what is meant here is in order at the outset. One can find publications that refer to any of the following as 'turbulence': (i) any process that increases structure at an interface, (ii) the early nonlinear behavior of an instability at interface, (iii) the development of substantially convoluted structure at an interface, having spectral content or spatial extent far beyond those of the initial state, (iv) the appearance of an inertial range in the fluctuation spectrum, with power-law decay of the spectral energy density, and (v) 


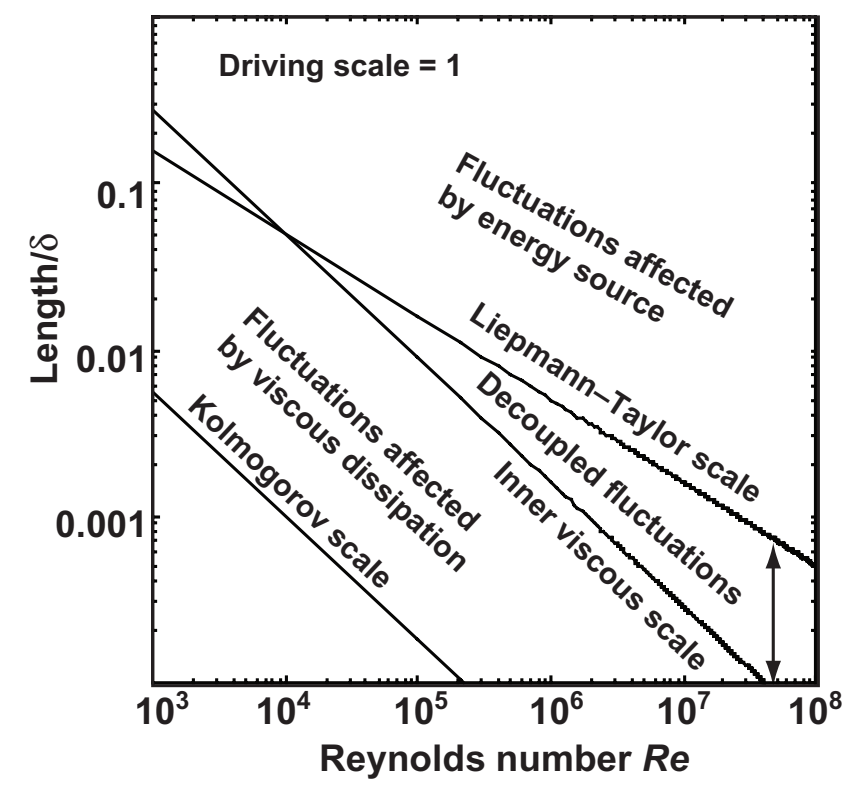

Figure 1. Some boundaries and regimes of fluctuation behavior are shown versus Reynolds number.

the development of strong mixing as indicated by supra-linear growth of the thickness of the mixing layer in time. Here our main interest is in processes that can lead to a mixing transition, in which fluctuations are present on a wide range of scales and quite thorough interpenetration of materials from the two sides of an interface is taking place. In a paper that will provide a useful reference point for our discussion, Dimotakis identifies the mixing transition as corresponding to item (iv) above, by analyzing the spatial scales present, and finds a minimum threshold Reynolds number for this transition. To set the context for further discussion, we briefly review the argument here.

Figure 1 shows some boundaries in a plot of normalized length versus Reynolds number $R e$, with the length normalized to the spatial scale on which structure is driven. This spatial, driving scale corresponds to the top edge of the figure. Over some range of sizes below this, the structure of the fluctuations present is directly affected by the energy source responsible for their existence. Below some spatial scale, however, one can expect that the spectral properties of the fluctuations will be determined primarily by their interactions with other fluctuations that do not differ greatly in scale. This limiting scale has some connection with the Taylor microscale, the largest physical scale on which the turbulent energy could possibly be dissipated by viscosity. Dimotakis identifies a spatial scale of this order as the Liepmann-Taylor scale, given by $5 \delta R e^{-1 / 2}$ and shown in the figure. Moving to the other end of the possible range of sizes, the Kolmogorov scale, given by $\delta R e^{-3 / 4}$, is the smallest spatial scale on which the turbulent energy present in the system can be dissipated. Energy in fluctuations on this scale is dissipated in one corresponding unit of hydrodynamic time. Fluctuations on a somewhat larger spatial scale than this are affected by viscous dissipation. Dimotakis chooses this region to correspond to a factor of 50 in size. This identifies the 'inner viscous scale', given by $50 \delta R e^{-3 / 4}$, with the notion that fluctuations on a larger spatial scale than this are not significantly affected by viscosity. As $R e$ increases, a range of spatial scales appears within which the fluctuations are decoupled both from the nature of the energy source and from viscous dissipation. It is this range within which energy-flow arguments like those of Kolmogorov [3] apply, so the appearance of this range should correspond to the development of an inertial range in the fluctuation spectrum. Dimotakis goes one step further and suggests that this also corresponds to the appearance of the mixing transition, supporting this suggestion by quite a few specific cases. This perspective, based on analysis of steady-state cases, provides a starting reference for discussions below.

It remains unclear how the turbulent spectrum comes to be full, so that the growing boundary layer encounters a continuously saturated spectrum of fluctuations. This is a fundamental idea in discussions of turbulence. (Dimotakis notes in a footnote that Kolmogorov [4] attributes it to Richardson.) Yet there is no general physical law which guarantees that saturated fluctuations will be present across a broad range of wavenumbers. We offer a speculation regarding this in section 5 .

We begin in section 2 with an overview of HEDP systems. We discuss how their production leads to their fundamental properties, and discuss the correspondence between HEDP systems and some astrophysical ones. In section 3, we introduce the HEDP fluid dynamics experiments, illustrating their key features. In section 4, we discuss specific instabilities that have been studied in the laboratory, showing how they develop differently in HEDP systems than in some other environments. There we draw a connection between a time-dependent analysis (by Zhou et al [35]) and the analysis of Dimotakis mentioned above. Motivated by some of the observations, we turn, in section 5 , to a more detailed discussion of the role of the $\mathrm{KH}$ instability relative to turbulence. Those with a previous understanding of HEDP systems are likely to find this section of the greatest interest. Section 6 concludes the paper.

\section{Properties of HEDP systems and correspondence to astrophysics}

There are a very wide range of existing or potential laboratory systems at high energy density. The systems of concern here are those in which substantial quantities of energy are delivered to a material target on ns timescales. In more detail, energies of at minimum hundreds of joules must be delivered on the time and space scales necessary to create energy densities above 0.1 TPascal $\left(10^{12} \mathrm{ergs}^{-3}\right)$, corresponding roughly to $\mathrm{mm}$ spatial scales and ns timescales, and increasing with the total energy. Other HEDP laboratory systems, not our focus here, include the delivery of $\mathrm{mJ}$ of energy to $\mu \mathrm{m}$ spatial scales by ultrafast (fs to ps) lasers, the potential creation of high-energy-density radiation environments on the forthcoming National Ignition Facility in the USA, and the environments found in nuclear quark-gluon plasmas.

The creation of our systems of interest typically involves the delivery to a surface of laser light or $\mathrm{x}$-rays (the latter generated by somehow heating high- $Z$ material). This generates a pressure at the surface of the order of 10 megabars, which drives a shock wave into the material. For a typical solid plastic material, having a density of about $1 \mathrm{~g} \mathrm{~cm}^{-3}$, 
the corresponding shock velocity is roughly $3 \times 10^{6} \mathrm{~cm} \mathrm{~s}^{-1}$, which is $30 \mathrm{~km} \mathrm{~s}^{-1}$. The sound speed in the material is of the order of $1 \mathrm{~km} \mathrm{~s}^{-1}$, so that the shock waves produced are typically of the order of Mach 30 . However, the sound speed is often rather uncertain because the 'preheating' of the material ahead of or 'upstream' of the shock wave by penetrating photons or electrons is often poorly known. On the one hand, the temperature of this upstream material may be somewhat smaller than $1 / 40 \mathrm{eV}$ (room temperature, $1 \mathrm{eV}=$ $11600 \mathrm{deg} \mathrm{K}$ ), because of radiative cooling of the target in vacuum before the experiment. On the other hand, if of the order of $0.1 \%$ of the initial energy from the laser or other source is deposited in the upstream material, then the temperature of this material can rise to of the order of $1 \mathrm{eV}$. Many experiments would show changes in their behavior if the upstream temperature were much larger than this, but experimenters have also been fooled more than once by preheat effects. It is worth noting that even when it does not affect the shock significantly, preheat can alter the initial conditions of structured interfaces [5].

It turns out that this uncertainty in Mach number, $M$, matters little for the fluid dynamics, because the compressible equations can be cast to involve terms scaling as $1 / M^{2}$. For example, the density jump produced by a shock wave can be written as

$$
\frac{\rho_{0}}{\rho_{1}}=\frac{(\gamma+1)}{(\gamma-1)+2 / M^{2}}
$$

for a polytropic gas of index $\gamma$. Here, the mass density is $\rho$ and the subscripts 0 and 1 are for upstream and downstream of the shock, respectively. For $\gamma \sim 1.5$, the impact of finite Mach number drops below $10 \%$ for $M=4.5$. (The index $\gamma$ is typically below the ideal-gas value of $5 / 3$ because of the degrees of freedom involved in ionization.) For HEDP media, which may have variable amounts of ionization, the post-shock temperature is

$$
k_{\mathrm{B}} T=\frac{A m_{\mathrm{p}}}{(1+Z)} \frac{2(\gamma-1)}{(\gamma+1)^{2}} u_{\mathrm{s}}^{2}
$$

in which $u_{\mathrm{s}}$ is the shock velocity, $A$ and $Z$ are the average atomic mass and ionization of the material, respectively, $T$ is the temperature, and $k_{\mathrm{B}}$ is the Boltzmann constant, given by $1.6 \times 10^{-12}$ for $T$ in $\mathrm{eV}$ and $\mathrm{cgs}$ units otherwise. For plastics or other low $-Z$ materials, $T$ is of the order of $5 \mathrm{eV}(\sim$ $50000 \mathrm{deg} \mathrm{K}$ ) for a $30 \mathrm{~km} \mathrm{~s}^{-1}$ shock, but note that it increases as the square of $u_{\mathrm{s}}$. HEDP materials in this regime are typically ionized from one to a few times, so that $1 \leqslant Z \leqslant 10$. Much higher temperatures and ionizations can be achieved in higher-atomic-mass materials, when they can be shocked at densities below solid density (in foams or gasses). Once the temperature becomes of the order of $100 \mathrm{eV}$ the transport of energy by radiation becomes an essential aspect of the dynamics and the shock wave becomes a radiative shock [2]. The actual transition to the radiative shock regime is quite abrupt but the transition temperature depends on specific details. Radiative shocks are beyond the scope of the present discussion.

A few details remain to complete the identification of key dimensional parameters for HEDP plasmas. The typical ion density is the density of ions in solids, of the order of $10^{23} \mathrm{~cm}^{-3}$, although many experiments work with
Table 1. Typical parameters in high-energy-density experiments.

\begin{tabular}{lccc}
\hline Parameter & Typical value & Low & High \\
\hline Flow velocity $U$ & $10 \mathrm{~km} \mathrm{~s}^{-1}$ & $1 \mathrm{~km} \mathrm{~s}^{-1}$ & $<1000 \mathrm{~km} \mathrm{~s}^{-1}$ \\
$\begin{array}{l}\text { Turbulent driving } \\
\text { scale } \delta\end{array}$ & $100 \mu \mathrm{m}$ & $10 \mu \mathrm{m}$ & $1 \mathrm{~mm}$ \\
$\begin{array}{c}\text { Kinematic } \\
\text { viscosity } v\end{array}$ & $10^{-5} \mathrm{~m}^{2} \mathrm{~s}^{-1}$ & $10^{-6} \mathrm{~m}^{2} \mathrm{~s}^{-1}$ & $10^{-4} \mathrm{~m}^{2} \mathrm{~s}^{-1}$ \\
$\begin{array}{c}\text { Reynolds } \\
\text { number } R e\end{array}$ & $10^{5}$ & $10^{4}$ & $10^{7}$ \\
\hline
\end{tabular}

densities some orders of magnitude smaller than this and inertial-fusion experiments seek to produce densities some orders of magnitude larger. The electron density is typically a few times the ion density, corresponding to the ionization. The material is dense and collisional enough that the ion and electron temperatures are tightly coupled and have the same value (for non-radiative shocks). Magnetic fields in the shock-heated matter, if present, are typically small enough to have no dynamic significance. Collisional magnetic diffusion times are in the range of a few ns. The collisional mean free path is short compared with the spatial scales of interest, so that using a fluid description rather than a particle description of the physical system is sensible.

Table 1 gives typical values of some of the parameters relevant to turbulence. The Reynolds number $R e$ is large, nearly always being above the approximate value of $10^{4}$ identified by Dimotakis [6] as the minimum for the mixing transition. Other parameters of interest are as follows. The Kolmogorov scale is typically of the order of $0.02 \mu \mathrm{m}$. The kinematic mass diffusion coefficient is of the order of the kinematic viscosity, so the Schmidt number is of the order of 1 . Heat flow, by both conduction and radiation, is typically negligible in comparison with convective energy transport [7], so that the corresponding dimensionless Peclet numbers are both large. One consequence of the above is that the Euler equations accurately describe the large-scale evolution of these physical systems. The limiting spatial scales of any turbulence are as always determined by viscosity. In addition, viscous effects are important in the dynamics of instabilities; with both the Rayleigh-Taylor (RT) and Richtmyer-Meshkov (RM) instabilities being damped for wavelengths below a few $\mu \mathrm{m}$ [8]. It is notable but likely accidental that this corresponds roughly to the Liepmann-Taylor scale discussed in section 1.

Connections to astrophysics arise readily because there are also numerous astrophysical systems whose large-scale behavior is accurately described by the Euler equations. These include supernovae, supernova remnants and the interactions of clumps or clouds with shocks or winds, among others. One consequence of this is that a well-designed laboratory experiment can provide a well-scaled model of a specific astrophysical process of interest, as was discussed by Ryutov et al [7]. Zhou [9] argues that to obtain detailed correspondence of the turbulent dynamics between an experiment and a typical astrophysical system with very high $R e$, the experiment must achieve a minimum $R e$ of $1.6 \times 10^{5}$. A second consequence is that one may learn things in the laboratory about the dynamics of strongly shocked, compressible systems that turn out to have applications in astrophysics. We will develop this correspondence further below. 


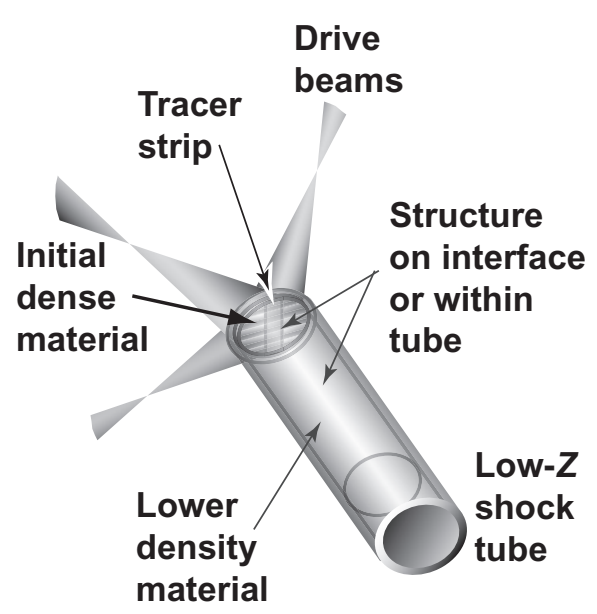

Figure 2. Generic sketch of experiment.

\section{HEDP fluid dynamics experiments}

As many readers of this topical issue will have little familiarity with HEDP, we discuss here the basic methods used in HEDP experiments to address issues in hydrodynamics or fluid dynamics. Figure 2 shows the essential elements of a typical experiment. There is an energy source that strikes a driven surface, represented here by three 'drive beams'. An actual laser experiment may use from one to dozens of drive beams. Alternatively, an experiment using a laser or using a type of device [10] known as a 'z-pinch' may irradiate the driven surface with $\mathrm{x}$-rays, typically having a thermal spectrum with a temperature of $100-300 \mathrm{eV}$. The driven surface is typically a low $-Z$ material and often a plastic, to avoid unnecessary radiation generation in the event that the second layer is a material of higher $Z$. The second layer is often the densest material in the target. The detailed reasons for this are complex, but have to do with maximizing the duration of the experiment without interference by unwanted shock waves or rarefactions. At times, these first two layers are of the same material.

Beyond these first two layers of material, the detailed structure of the target is determined by the experiment. The second layer may include a tracer strip, as indicated, designed to match the layer in hydrodynamic behavior but to include a high- $Z$ dopant that will preferentially absorb diagnostic $\mathrm{X}$-rays. The second layer may end at a structured interface, and certainly does so for studies of the RT and/or RM instabilities. In these cases and others observing interface evolution, there is a third layer of lower density material, as indicated in the figure. This material is often a cellular foam, such as an aerogel, which to date has been the only practical way to introduce densities of tens to hundreds of $\mathrm{mg} \mathrm{cm}^{-3}$ into these targets. A lower density third layer of material also may be of use if a faster shock is needed than the one produced naturally by the energy source. In other cases, the second layer of material may extend throughout the target, and structures intended to interact with the shock wave might be placed within it or even beside it. The target typically includes shields or other structures required by diagnostics, in addition to features required for alignment or for spatial calibration of diagnostics, none of which are shown here.
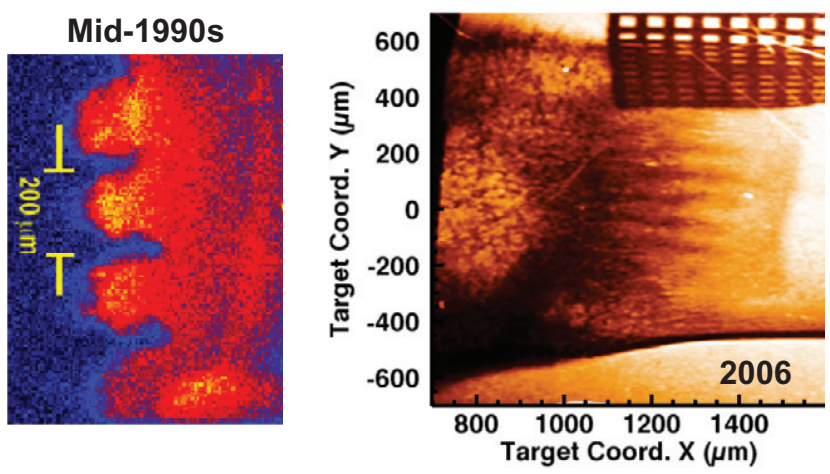

Figure 3. Radiographic images from the mid-1990s (left) and from 2006 (right).

The workhorse diagnostic for hydrodynamic HEDP experiments is x-ray radiography. One uses an additional energy source-additional laser beams in a laser experiment- to irradiate a target designed to produce the x-rays of interest. Current experiments most often irradiate a thin layer of material to produce radiation in the 'Helium-alpha' line(s), which are the $n=1-2$ transitions from a mid- $Z$ material ionized into a helium-like state. Under properly chosen conditions, this radiation dominates the emission spectrum, permitting the use of relatively simple filters in front of the detector to select a spectral band. The x-ray energies obtainable by this method range from 1.5 to $10 \mathrm{keV}$. Advances in technique have greatly improved the resolution and have greatly decreased the noise in images obtained this way, in part by developing ungated imaging directly onto x-ray film, without any intervening (and noisy) detection or gating elements. It is now possible to obtain two simultaneous orthogonal images by these techniques [11]. Figure 3 illustrates the progress that has been made, comparing a radiograph from the mid-1990s to one obtained in December 2006. In both cases, a laser-generated shock front, followed by rarefaction, first shocks and then decelerates and interface that is initially imprinted with a small-amplitude pattern. The RM and RT instabilities lead to the outward penetration of spikes of denser material and the relative inward penetration of bubbles of less-dense material. In the earlier image, one can see the spikes of dense material, but cannot see much detail and can barely make out the shock wave propagating to the right. In the later image, one can see the detailed structure within the unstable material, can see the shock clearly, and can see very well the $43-\mu \mathrm{m}$ openings in the calibration grid. Other radiographic diagnostics are also sometimes used. Two examples include the use of a spherical imaging crystal to form a monochromatic image [12] and the use of a very intense, ultrafast laser to produce higher energy $\mathrm{x}$-ray sources [13].

\section{Specific instabilities in the laboratory and astrophysics}

We now proceed to address the three iconic primary instabilities: RM, RT and KH. In each case, we will discuss how these processes are produced in experiments, will show some experimental data and will discuss the advent of 


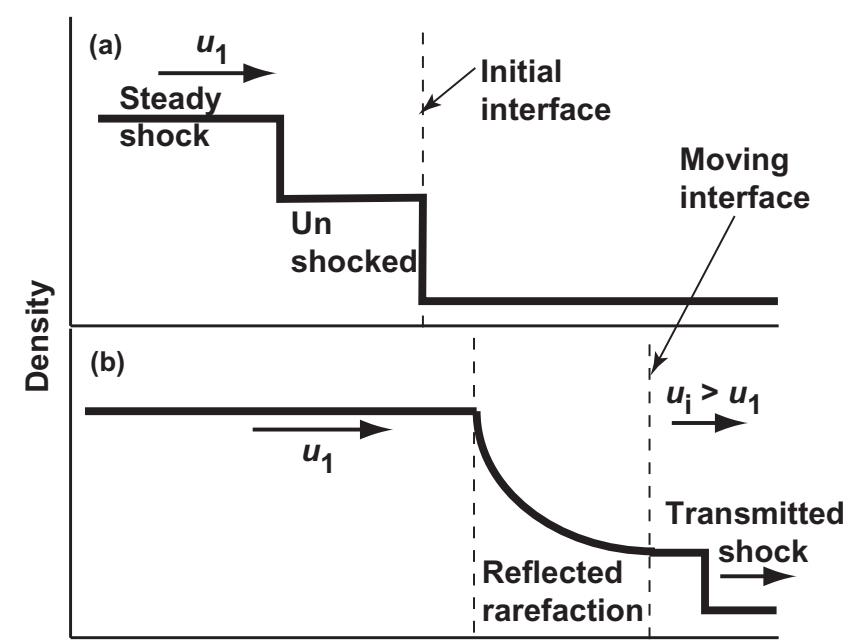

Position in lab frame

Figure 4. 1D development of RM experiment.

turbulence in that case. We also draw some connections with astrophysics.

\section{1. $R M$}

The RM process is most often defined as the growth of structure at an interface that initially is not planar, following the passage of a shock wave through it. More generally, it involves the growth of structure due to transverse velocity perturbations at an interface, however they may be generated [14]. RM is generally referred to as an instability, although this is not technically an accurate description [2]. In HEDP experiments, one can most readily produce RM by driving a steady shock through a structured interface. In principle, one has the choice whether to drive the shock through a dense layer into a less-dense layer or vice versa. In practice, the issue of experiment duration and wave speeds makes the dense-to-less-dense case the only practical choice.

Figure 4 illustrates the resulting one-dimensional (1D) dynamics, by showing the spatial profile of the density. Once the incoming shock wave overtakes the interface, the interface begins moving at a speed $u_{\mathrm{i}}$ and a transmitted shock moves through the less-dense material. Simultaneously, a reflected rarefaction moves backward, in a Lagrangian sense, through the incoming, shocked, dense material. The dynamics near the interface, in both this RM case and in analogous RT cases, develops at subsonic speeds in the post-shock plasma environment. As a result, incompressible theory is a reasonable model for this aspect of the system evolution. In an experiment, the initial interface is rippled or otherwise has structure, so that the passage of the shock creates transverse velocity perturbations at the interface. (Alternatively, one can say that the shock deposits vorticity at the interface.) These transverse perturbations lead the interface to become more structured with time. In traditional experiments and simulations at low Mach number, the shock wave moves far from the interface and RM produces spikes whose tips broaden and roll up due to the lift generated as they divert the material they penetrate (a process often described as ' $\mathrm{KH}$ roll up'). Turbulence then may develop within these rollups.
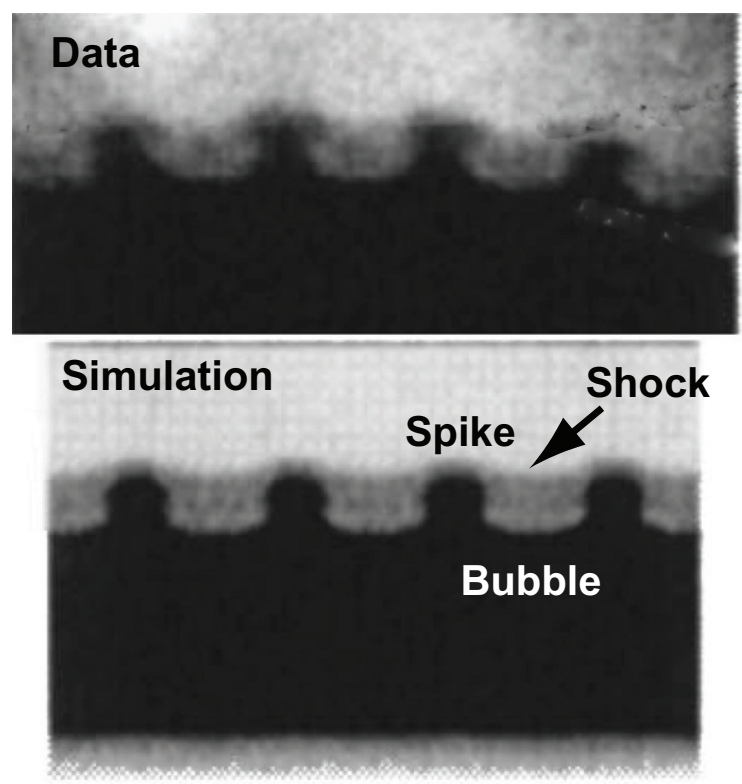

Figure 5. A high-Mach-number RM experiment, adapted from Glendinning et al [15].

This does not occur in RM at high Mach number for the following reason. A standard expression for the rate of increase of a sinusoidal perturbation of amplitude $\eta$, attributed to Meyer and Blewett, is

$$
\frac{\mathrm{d} \eta}{\mathrm{d} t}=A \frac{k\left(\eta_{0}+\eta_{1}\right)}{2} u_{\mathrm{i}},
$$

where $A$ is the post-shock Atwood number and $k$ is the wavenumber of the perturbation. In the strong shock limit, the speed at which the shock separates from the interface is

$$
u_{\mathrm{sT}}-u_{\mathrm{i}}=\frac{\gamma-1}{2} u_{\mathrm{i}}
$$

in which the transmitted shock speed is $u_{\mathrm{sT}}$. In systems with strong shocks, the growth rate of equation (3) can easily exceed the separation speed of equation (4). In this case, the spikes push against the shock, distorting it and slowing their own growth. This phenomenon was most clearly observed in experiments by Glendinning et al [15], whose results are shown in figure 5 .

RM certainly occurs in high-Mach-number astrophysical contexts. For example, in supernova remnants the stellar ejecta drive a nearly steady shock through the circumstellar medium for an extended period of time. This is a very strong shock. One would expect the effects of RM to be present and to increase the structure in the medium after the passage of the shock through density changes. One paper has explored this [16], finding that such effects are present but also that they fade with time, under standard assumptions, as the system evolves.

When RM is present in either HEDP experiments or astrophysical systems driven by strong shocks, one must conclude that it is a poor candidate to produce turbulence in any sense related to a mixing transition or even to producing significantly convoluted structures. There might be some potential that perturbations of initially very small amplitude, for which RM did not overtake the shock, could develop such 
a structure. In the laboratory at least these would be hard to see. In addition, it is well known that, once a second shock wave passes through the structure generated by RM, very complex fluctuations develop.

\section{2. $R T$}

The simple version of the RT instability occurs when a less dense fluid supports a more dense fluid against gravity or some other acceleration. More generally, RT occurs whenever the gradients of pressure and density are opposed, and also in cases having a sufficiently steep pressure gradient in comparison with the density gradient [17]. The evolution of this instability into a turbulent state has long been an active area of study. Two examples, out of a large body of work, are experimental [18] and simulation [19] papers by Dimonte et al. RT can enter a turbulent state by at least two paths. In the work just cited, a multimode initial condition leads to a competition between modes, in which longer wavelength modes with higher nonlinear terminal velocities overtake shorter wavelength modes that initially grow quickly, a regime known as the bubble-merger regime [20, 21]. Alternatively, RT from a single-mode initial condition can develop spikes whose tips broaden and roll up (just as those for RM do), after which instabilities within the tip rolls initiate a turbulent transition [22]. A further possibility, seen only in simulations to date, is that broadened RT spike tips may interact to initiate a turbulent transition [23]. In addition, in the event that the spike tips do not greatly broaden, the shear flow along the boundary between the spikes and the bubbles seems a likely source of $\mathrm{KH}$ instabilities that also might lead to turbulence. Simulations using some numerical schemes show such structures [24, 25], although the numerical Reynolds number is small enough in these simulations that they cannot indicate whether a turbulent transition occurs. We discuss this specific issue further in the next section.

The RT instability has been observed in HEDP systems, both at the ablation surface [26], where the action of the energy source causes hot, low-density plasma to accelerate cooler, denser plasma, and at an embedded interface [27], a case closer to the simple RT case. Other extensive work has also been undertaken and is ongoing in the context of inertial fusion research. In addition, RT growth has been studied when a blast wave first shocks and then decelerates an embedded interface, in experiments motivated by supernova explosion dynamics [28-31]. In these cases, there is an initial $\mathrm{RM}$ response followed by a longer period of RT growth. In published work, however, none of these RT experiments have entered a turbulent phase in the sense of cases (iv) or (v) in section 1 .

As an example, the radiograph on the right in figure 4 is from one of the experiments with a blast-wave-driven interface. In this specific case, the interface had a 3D initial perturbation including an egg-crate-like pattern made up of sinusoidal perturbations having $71-\mu \mathrm{m}$ wavelengths in two orthogonal directions and a peak amplitude of $2.5 \mu \mathrm{m}$ combined with a sinusoidal perturbation in one of these directions having a 424- $\mu \mathrm{m}$ wavelength and a peak amplitude of $2.5 \mu \mathrm{m}$. The data are from $25 \mathrm{~ns}$ into the experiment. The target included a tracer strip, as discussed above, so that the

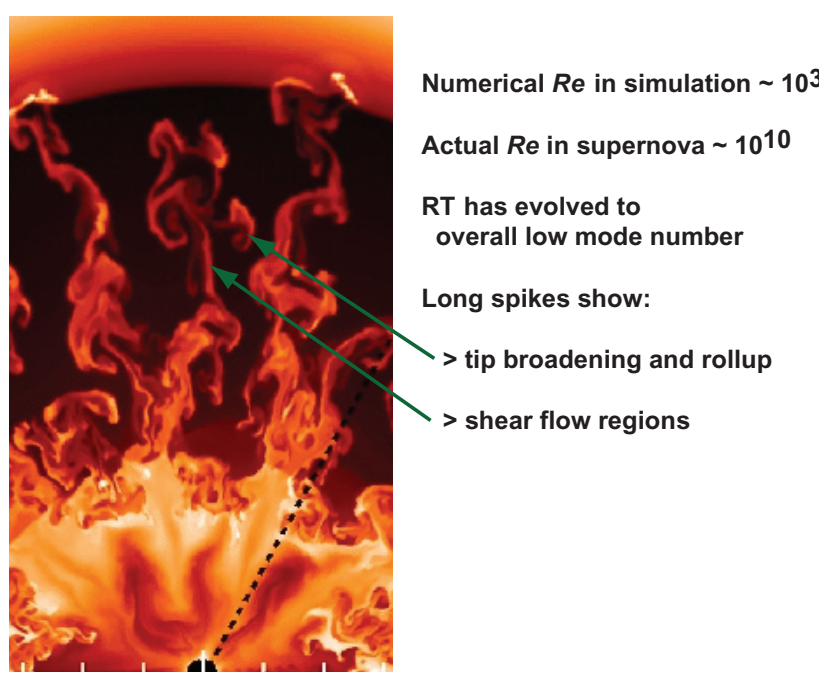

Figure 6. A color display of the logarithm of density from a simulation of Supernova 1987A, adapted from Kifonidis et al. One can see both tip rollups and shear layers that in principle might be sites of a transition to turbulence, were the Reynolds number larger.

intensity variations in the image are primarily due to structure near the mid-plane of the shock tube. The bubbles and spikes are about $250 \mu \mathrm{m}$ long, having grown at an average of about $10 \mu \mathrm{m} \mathrm{ns}^{-1}\left(10 \mathrm{~km} \mathrm{~s}^{-1}\right)$ throughout the experiment. Using this velocity, a transverse dimension of $100 \mu \mathrm{m}$ and a kinematic viscosity of $10^{6} \mu \mathrm{m}^{2} \mathrm{~s}^{-1}$, one finds $R$ to be $10^{6}$. Note also that in this case, there are no indications of rollups at the spike tips. Experiments using 2D initial perturbations, having a comparable value of $R$, do observe definite rollups at the tips but also do not show an onset of turbulence [29], in contrast with results from low-energy-density, low-Atwood-number experiments [22].

The laser experiments just discussed were performed with the intention that they would be relevant to the explosion phase of supernovae. Both the experiment and a supernova have a large Reynolds number, a large Peclet number, and small radiative energy transport. Both involve a very strong shock and ionized post-shock plasma. Both systems are sensibly described by the Euler equations. In both cases, the passage of a blast wave produces a large-Atwood-number interface that decelerates subsequently. As a result, the experiment is a good model of a small patch of the star during the explosion phase, until the finite lateral size of the experiment begins to affect its behavior. This corresponds to a few tens of ns in the experiment and a few thousand seconds in the stellar explosion. The comparison of the experiment with simulations of supernovae would be of great interest if the experimental system were to undergo a mixing transition, which is precluded in the simulation by its low numerical Reynolds number. Figure 6 shows results of a simulation of Supernova 1987A by Kifonidis et al [32], in order to illustrate that a turbulent transition seems likely. It requires very special initial conditions [33] to produce the outward motion of inner material observed in Supernova 1987A. The specific simulation shown, like most others, did not produce enough motion. It is unclear at present whether a mixing transition would increase or decrease the outward motion.

In the context of the picture developed by Dimotakis, the above results are all puzzling. The laboratory experiments 
all have $R e$ above $10^{5}$, and the astrophysical systems have values of $R e$ that are orders of magnitude larger. Yet the laboratory experiments do not produce a mixing transition. It is harder to tell in the astrophysical cases, but there is also no strong evidence that such a transition has occurred. Observationally, various elements do seem to remain in distinct clumps. This raises the question of why such systems have not become turbulent. An answer to this question has been provided in the papers by Robey et al [34] and Zhou et al [35]. The Zhou-Robey hypothesis is that the uncoupled fluctuations develop within laminar-flow regions established in the boundary layers created by viscous diffusion at locations of significant shear, and that, in order for a turbulent transition to occur, these boundary layers must grow for a long enough time that their extent exceeds the inner viscous scale. In these papers, this hypothesis is shown to explain the onset of turbulence in several specific cases. The hypothesis also explains the absence of turbulence in the blast-wave-driven instability experiments discussed above - the experiments did not endure long enough.

Mathematically, the Zhou-Robey hypothesis states that there are two necessary conditions for the transition to turbulence. The first is that of Dimotakis discussed above, that the Liepmann-Taylor scale, $\lambda_{\mathrm{LT}}=5 \delta R e^{-1 / 2}$, be greater than the inner viscous scale, $\lambda_{\mathrm{v}}=50 \delta R e^{-3 / 4}$. The second is that the viscous boundary layer thickness, $\lambda_{\mathrm{b}}=5(\mathrm{nt})^{1 / 2}$, exceed the inner viscous scale $\lambda_{\mathrm{v}}$. There is an explicit time dependence in $\lambda_{\mathrm{b}}$, but the Reynolds number may also be time-dependent, as it is in systems where the scale of the turbulent layer increases with time. Even so, one typically finds that the viscous boundaries take longer to exceed the inner viscous scale than $R$ does to reach $\sim 10^{4}$ where $\lambda_{\text {LT }}$ exceeds $\lambda_{\mathrm{V}}$.

Here, we would like to offer an observation that connects these two conditions with a well-known aspect of turbulent systems. If one solves the Zhou-Robey condition for turbulent onset, $\lambda_{\mathrm{b}}=\lambda_{\mathrm{v}}$, for $t$, one finds

$$
t=\frac{100}{\sqrt{R}} \frac{\delta}{U} .
$$

For typical turbulence, $\delta / U \sim d / u$, where the eddy scale is $d$ and the turbulent velocity scale is $u$, and the ratio $d / u$ is the scale of the eddy-turnover time. Thus, one sees that for $R$ near its threshold value of $10^{4}, t$ is near one eddy-turnover time. This is consistent with the experimental observation that large turbulent eddies are typically seen to dissipate through turbulence in about one eddy-turnover time. Another way to state this point is to say that when $R$ is near its threshold value, an eddy-turnover time is required to initiate the turbulent mixing transition.

\section{3. $\mathrm{KH}$}

The KH instability develops in the presence of velocity shear for which small perturbations produce lift forces that tend to increase the size of the perturbations. It is only a semantic point whether or not one describes all lift-generated responses, such as spike-tip broadening in RM or RT, as KH, or whether one limits the use of $\mathrm{KH}$ to unstable growth at extended shear layers. $\mathrm{KH}$ plays a role in transitions to turbulence that involve the initial growth and saturation of structure at shear layers. After this, secondary instabilities from the initial condition established by $\mathrm{KH}$ initiate the growth of further structure and the subsequent, explosive amplification of vorticity [36, 37].

To set some context for the discussion that follows, we first review some aspects of KH theory. The theory of the KH instability begins in all cases with the equations describing a fluid in motion (or with the magnetohydrodynamic (MHD) equations for magnetized systems). For the unmagnetized case, without gravity or surface tension, these are

$$
\begin{gathered}
\rho \frac{\partial u_{i}}{\partial t}+\rho u_{k} \frac{\partial}{\partial x_{k}} u_{i}=-\frac{\partial}{\partial x_{i}} p+\frac{\partial}{\partial x_{k}} p_{i k} \\
\frac{\partial \rho}{\partial t}+\frac{\partial}{\partial x_{k}}\left(\rho u_{k}\right)=0 .
\end{gathered}
$$

Here we sum over repeated indices and follow the notation of Chandrasekhar [38], with time $t$, components of displacement $x_{i}$, density $\rho$, fluid velocity components $u_{i}$, scalar pressure $p$ and viscous stress tensor $p_{i k}$ given by

$$
p_{i k}=\rho v\left(\frac{\partial u_{k}}{\partial x_{i}}+\frac{\partial u_{i}}{\partial x_{k}}\right)
$$

in which $v$ is the kinematic viscosity. In addition, one takes $\nabla \cdot \mathbf{u}=0$ if the fluid is incompressible. Further details are discussed in the standard references and do not bear repeating here. The equation of the interface, the jump conditions across the interface, and the boundary conditions also may be required to develop a solution. By analyzing perturbations about an initial state that specifies the density, flow velocity and kinematic viscosity in the fluid on each side of the interface, one can seek the growth rate of unstable modulations of the interface. Throughout this discussion, we will ignore surface tension and other effects that might complicate specific cases. We also ignore gravity, even in systems with RT-produced shear layers. This is to some degree justified as the strongest shear layers, between bubbles and spikes, admit modulations that are perpendicular to gravity. Of course, the detailed response of the entire system will involve the coupled RT and $\mathrm{KH}$ responses, which will have to be calculated numerically. Nonetheless, we will see that an evaluation of the isolated $\mathrm{KH}$ response proves to be informative.

For the simplest case, in which two uniform incompressible fluids, flowing at different velocities in the same $(x)$ direction, meet at a boundary, the KH growth rate is found to be

$$
\gamma=k_{x} \Delta U \frac{1}{2} \sqrt{1-A^{2}}
$$

in which $k_{x}$ is the $x$-component of the wavevector (along the direction of flow), $\Delta U$ is the difference in velocity between the two fluids, and $A$ is the Atwood number of the interface, given by $A=\left(\rho_{1}-\rho_{2}\right) /\left(\rho_{1}+\rho_{2}\right)$, where $\rho_{1}$ and $\rho_{2}$ are the densities of the two fluids.

If the two fluids are compressible but the problem is otherwise unchanged, then Gerwin [39] shows that the dispersion relation is

$$
F(\phi)=\phi^{2} \sqrt{(\phi-M)^{2}-1}-(\phi-M)^{2} \sqrt{\phi^{2}-1}=0
$$




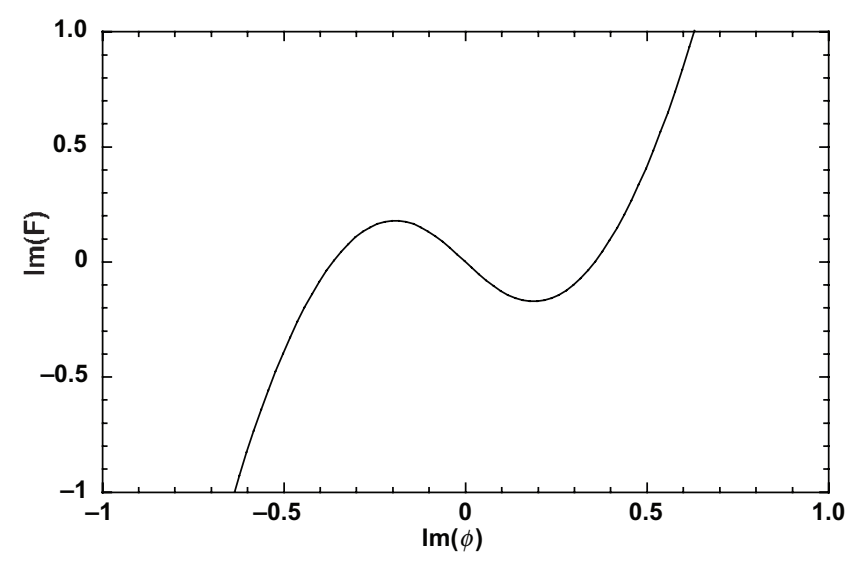

Figure 7. The dependence of $\operatorname{Im}(F)$ on $\operatorname{Im}[\phi]$, for $\operatorname{Re}(\phi)=M / 2$, which makes $\operatorname{Re}[F]=0$. One of the two imaginary roots is unstable.

in which $\phi$ is the frequency (with the growth rate being the imaginary part), normalized by $k s$, and $M$ is an effective Mach number, $k_{x} \Delta U /(k s)$. Here, $k$ is the total wavenumber and $s$ is the sound speed. Note that the ratio $\operatorname{Im}(\phi) / M$ equals $\gamma /\left(k_{x} \Delta U\right)$. The effective Mach number has a maximum value of $\sqrt{8}$, so that at large flow velocities only sufficiently oblique waves are unstable. The roots of interest of this equation occur when $\operatorname{Re}(\phi)=M / 2$, so that $\operatorname{Re}(\mathrm{F})=0$. Figure 7 shows the dependence of $\operatorname{Im}(F)$ on $\operatorname{Im}(\phi)$ for this case, for $M=2.5$. The curves shown are typical for $1<M<\sqrt{8}$. The roots occur near $\operatorname{Im}(\phi)=0.5$, so that $\gamma /\left(k_{x} \Delta U\right)$ is in the range of $0.2-0.5$, not unlike the incompressible case. In the following, we will not explicitly discuss compressible results. We will make the reasonable assumption that the growth rates in such cases are similar enough to those in incompressible systems that the arguments we make apply to both. There may be some exceptions, but their identification remains for future work. It is also often the case in HEDP experiments that the mixing layer is at nearly constant pressure and its growth is subsonic, so it would be sensible to apply an incompressible theory in any event.

Turning to HEDP experiments, $\mathrm{KH}$ has been remarkable by its absence. Firstly, consider the RT experiments described above. The size of the mixing layer containing the bubble and spikes increased at $10 \mathrm{~km} \mathrm{~s}^{-1}$. About half of this was stretching due to the rarefaction that followed the blast wave (similar to that illustrated in figure 4). Using $5 \mathrm{~km} \mathrm{~s}^{-1}$ as the effective incompressible shear velocity and at Atwood number of 0.8 , one finds from equation (9) that a structure of $10 \mu \mathrm{m}$ wavelength along the spike/bubble interface would have a growth rate of nearly $1 \mathrm{e}$-foldings per ns. If such a growth had occurred, then the atomic-scale structure would have long since grown to saturation amplitude before the time of the image in figure 4 . In contrast, in 3D simulations of $\mathrm{RT}$, initialized by a 2D sinusoidal perturbation and subject to constant acceleration, Calder et al [25] found substantial growth of the structure along the spike/bubble shear layer, as figure 8 shows. This calculation used the purely Eulerian FLASH code.

Other HEDP experiments have explored supersonic jets that penetrate another medium. These experiments typically shock a layer of material that then accelerates and expands down an evacuated channel, forming a supersonic directed flow that then emerges into another material. Figure 9 shows an example, from work by Foster et al [40]. In this case, a Ti jet emerged into low- $Z$ foam. The radiographic image was taken at $200 \mathrm{~ns}$. This jet has $R \sim 10^{6}$ and flows at about $10 \mathrm{~km} \mathrm{~s}^{-1}$ to the right through a nearly stationary volume of shocked foam plasma. The jet density is near the foam density for much of the expansion, so the Atwood number is small. The corresponding linear $\mathrm{KH}$ growth rate, for modes of 100 - $\mu \mathrm{m}$ wavelength, is $\sim 0.3$ per ns. Yet even earlier in time, there are no signs in the data of $\mathrm{KH}$ growth along the edges of the jet. This contrasts sharply with the subsonic jets in fluids discussed, for example, by Dimotakis [6, 41].

A similar circumstance exists with regard to astrophysical jets. These jets remain collimated over large distances and do not exhibit the development of increasingly small-scale structures or the lateral expansion of the jet material that would correspond to the onset of turbulence at the shear layer along the edge of the jet. Even so, astrophysical simulators, running codes based on the Euler equations, have found, beginning with a classic paper by Blondin et al [42], that these jets should be KH-unstable. A large number of subsequent papers have explored jet structure under various assumptions, including, for example the effect of $\mathrm{KH}$ in 3D [43], the effects of pulsations in the jet source combined with magnetic fields [44, 45], and the consequences of different types of cooling [46]. Such simulations do not easily obtain sufficient resolution to resolve the details [47, 48]. For a jet from a young star, a typical jet velocity is $100 \mathrm{~km} \mathrm{~s}^{-1}$. The Atwood number varies, we assume 0.9 here. A typical jet radius is of the order of $10^{10} \mathrm{~km}$. For a wavelength of $10^{16} \mathrm{~cm}$, the corresponding $\mathrm{KH}$ e-folding time from equation (9) is of the order of 100 years. This is within an order of magnitude of the values found in more realistic theory including radiative and other effects [49] (converting spatial growth rates into temporal ones by multiplying the spatial growth rate by the jet speed divided by its radius). These jets propagate for tens of thousands of years. Given the large value of $R$ and the large number of $\mathrm{KH}$ growth times over which these jets have endured, however, it is difficult to understand why these jets have not become turbulent, and do not exhibit the corresponding strong lateral transport of material that would result.

In the following section, we suggest a solution to the puzzle that so many shear flows, though predicted to be $\mathrm{KH}$ unstable, show little evidence of a transition to turbulence. With regard to experiments, the observations just discussed have increased the significance of experiments aimed at directly producing $\mathrm{KH}$ in supersonic HEDP systems. A design for such an experiment, in which a propagating shock creates a system that is $\mathrm{KH}$ unstable by flowing rapidly along a modulated interface to a higher density material, is given in [50]. Another experiment (by E C Harding and collaborators) that creates a flowing plasma that then encounters and flows along a modulated interface is also under way.

\section{Role of KH in turbulent onset}

Two aspects of real systems are missing from the theory discussed above: velocity gradients and viscous diffusion, 


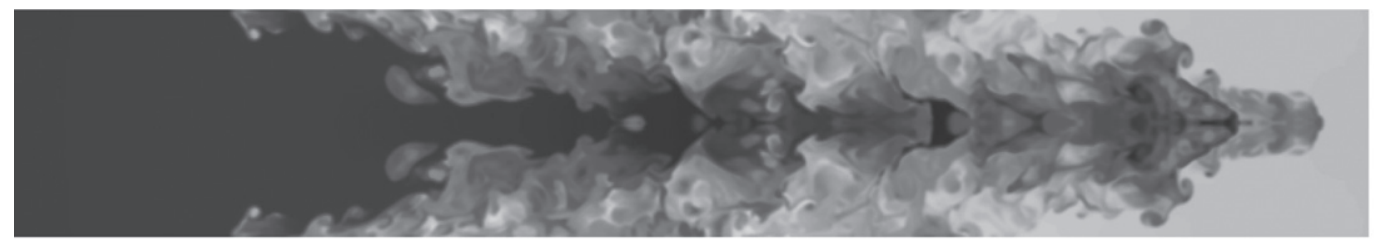

Figure 8. Density display from a single RT spike calculation using the code FLASH. This case used 128 resolution elements vertically, and periodic boundary conditions at the vertical boundaries. Adapted from Calder et al [25].

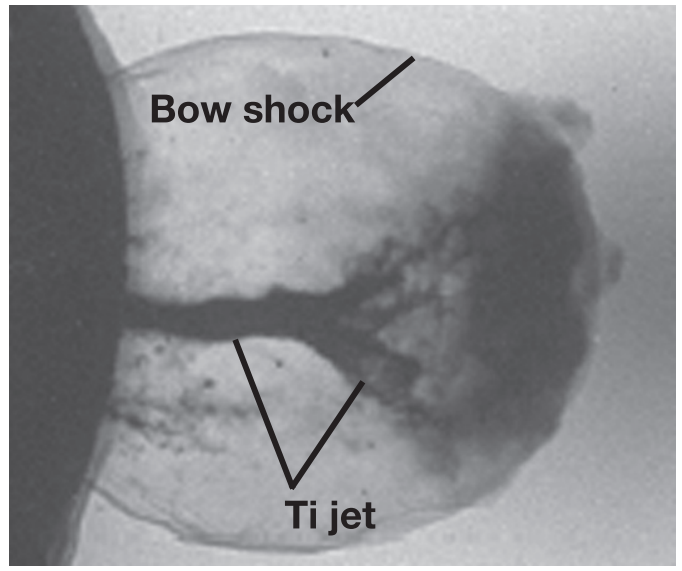

Figure 9. Supersonic jets. (a) From a laboratory experiment (adapted from Foster et al [40]).

although diffusion does play a role in the Zhou-Robey hypothesis. These effects are linked, as viscous diffusion acts to weaken the gradient in velocity across a shear layer. The simulations discussed above in principle include velocity gradients, but in actuality often do not model the process by which a shear layer forms and also do not accurately treat viscous diffusion. This can limit the existence of $\mathrm{KH}$ or its ability to contribute to turbulent fluctuations. We discuss the incompressible case, which should be approximately accurate for the reasons discussed above.

For systems that evolve in time, the free shear layer thickness is approximately $H=H_{0}+\sqrt{v t}$, in which $v$ is the kinematic viscosity, $t$ is time, and $H_{0}$ is the initial boundary layer thickness established as it forms. The fact that $H_{0}$ is finite reflects that the formation of the shear layer, and experimental effects such as preheating that may occur prior to shear layer formation, can limit the steepness of the velocity gradient. This will be quite important later in our discussion. Esch [51] provides a thorough discussion of $\mathrm{KH}$ in the presence of broadened shear layers, including both analytical and numerical results and consideration of different profiles across the layer. In this regime of large $R$ it is not the viscous dissipation that directly limits $\mathrm{KH}$, but rather it is the presence and impact of the boundary layer. For large $R$, the results of Esch are substantially the same as those obtained from the simpler model of Chandrasekhar [38], which we discuss here. This treatment assumes a linear transition in velocity and density between the two fluids and examines the fastest growing modes, which have $k=k_{x}$. If the (full) transition distance is $H$, then the normalized wavenumber is $\kappa=k H$ and the normalized frequency is $n=2 \gamma / k_{x} \Delta U$. With these definitions, the dispersion relation is

$$
\begin{aligned}
& e^{-2 \kappa}\left[1+\frac{1}{2} A \kappa(n+1)\right]\left[1+\frac{1}{2} A \kappa(n-1)\right] \\
& -\left[1+\kappa(n+1)\left(\frac{1}{2} A-1\right)\right]\left[1+\kappa(n-1)\left(\frac{1}{2} A+1\right)\right]=0 .
\end{aligned}
$$

We want to explore this somewhat as we will use an approximation to this result in our further calculations below. One can, for example, fix $A$ and solve this equation for the dependence of the growth rate on wavenumber. This also allows one to find the maximum unstable wavenumber, $k_{\mathrm{th}}$. Figure 10 compares the solutions to this dispersion relation with those of the $H=0$ result discussed above, for $A=0.3$, 0.6 and 0.9 . The results for $A<0.3$ change little.

Two observations about figure 10 will support our further analysis. Firstly, the threshold wavenumber is approximately given by $k=1.4$. This is comparable with the result of Esch mentioned above and corresponds to a wavelength of $\lambda \sim 5 H$. Secondly, it is not a bad approximation to take

$$
\gamma /\left(k_{x} \Delta U\right)=\frac{1}{2} \sqrt{1-A^{2}}\left[1-\frac{k}{k_{\mathrm{th}}}\right]
$$

which typically will produce errors less than a factor of two. We will use this below.

As a first application of this result, we note that $H_{0}$ may at times be a significant fraction of $\delta$. Some simulations indicate that this may be the case for RT under conditions similar to those of figure 4 (taking $\delta$ to be the spike width). In addition, the production of jets like those shown in figure 9 by launching material through a tube is likely to produce a significant radial velocity gradient within the jet material, thus reducing the velocity gradient between the jet material and the shocked foam. And while we have much less understanding of jet formation in astrophysics, it is certainly possible that the velocity gradients there will be less steep than has been assumed in some of the simulations.

We now turn to a speculation regarding the potential role of $\mathrm{KH}$ in the development of turbulence at smaller scales. It may be that the secondary instabilities or the mode coupling of large-scale fluctuations manage to fully populate the fluctuation spectrum at all scales down to the inner viscous scale. But it may also be that $\mathrm{KH}$ has an important role to play in developing the fluctuations at newly formed small-scale shear layers. We explore that here.

At the smallest scales, there is a competition between $\mathrm{KH}$ growth and the growth of the boundary layer between the fluids. Modulations at some specific $k$ will grow in time 


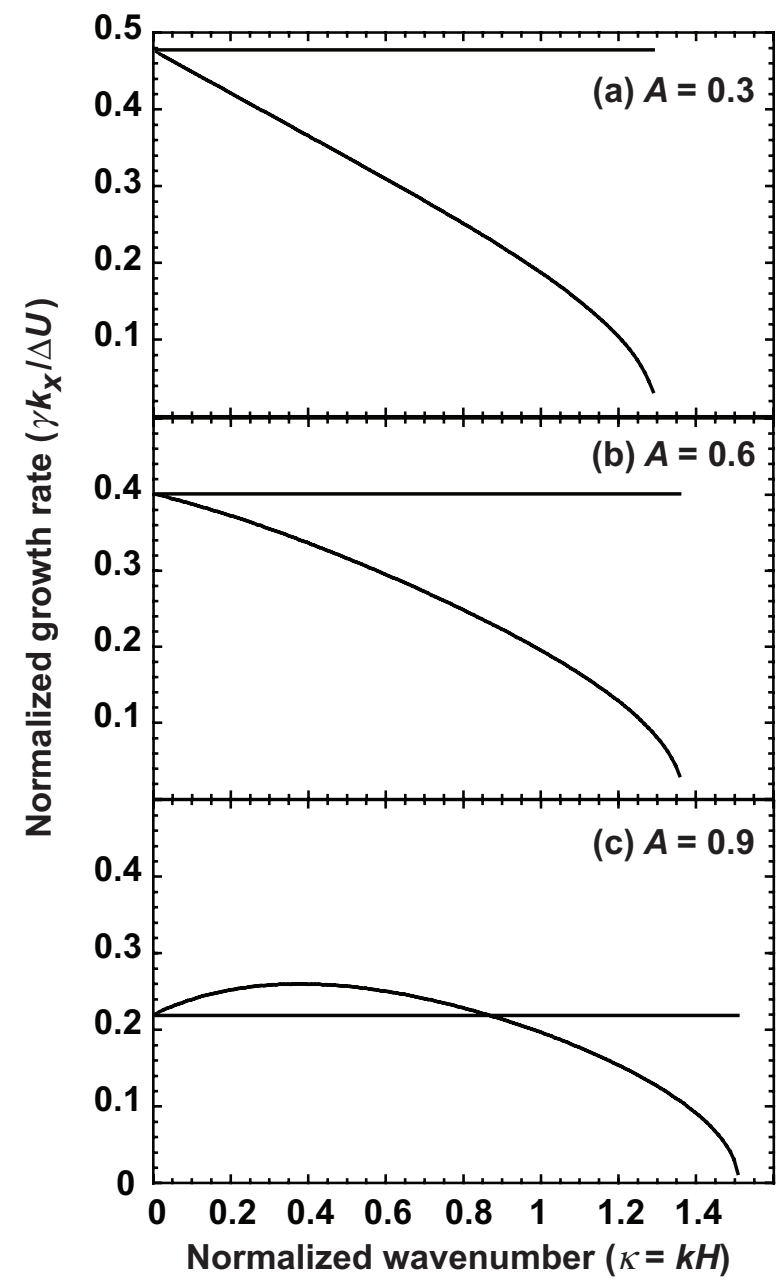

Figure 10. Dependence of normalized growth rate, $\gamma /\left(k_{x} \Delta U\right)$, on normalized wavenumber, $\kappa=k H$, for a boundary layer of thickness $H$. The straight line is the $H=0$ result in each case. (a) $A=0.3$, (b) $A=0.6$ and (c) $A=0.9$.

until the boundary layer becomes thick enough that $k H \sim 1.4$. The interesting question is: How much growth will there be? This will determine whether $\mathrm{KH}$ can become large enough to enable the secondary instabilities to generate the transition to turbulence. The calculation of this growth proceeds as follows. We have $k_{\mathrm{th}}=1.4 / H$. This implies that the time, $t_{1}$, when $k_{\text {th }}=k$, is given by

$$
t_{1}=\frac{1}{v}\left(\frac{1.4}{k}\right)^{2}\left(1-\frac{k H_{0}}{1.4}\right)^{2}
$$

We can find the number of e-foldings by which the initial modulations will be amplified, for $k=k_{x}$ as

$$
\begin{aligned}
\int_{0}^{t_{1}} \gamma \mathrm{d} t & =\int_{0}^{t_{1}} k \Delta U \frac{1}{2} \sqrt{1-A^{2}}\left[1-\frac{k}{k_{\mathrm{th}}}\right] \mathrm{d} t \\
& =\left(\frac{\delta \Delta U}{v}\right) \frac{1}{3 k \delta} \sqrt{1-A^{2}}\left(1-k \delta \frac{H_{0}}{1.4 \delta}\right)^{3} .
\end{aligned}
$$

We can use equation (9) to establish a saturation scale for comparison with the other scales involved in the mixing transition. Firstly, we must establish some number of e-foldings of amplification that we assume corresponds to the onset of secondary instabilities and then turbulence.

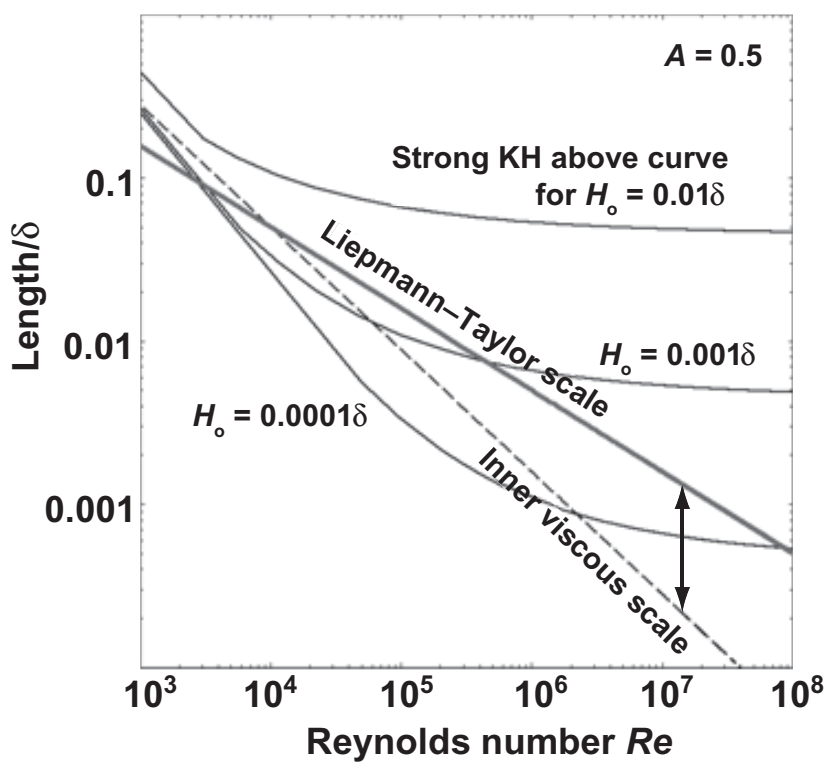

Figure 11. Impact of initial shear-layer transition width.

The fluctuations that must be saturated include those with $k=k_{x}$, described by equation (6) and also those with $k>k_{x}$, which have somewhat less amplification. Here, we will use 10 e-foldings for $k=k_{x}$ to approximate this level of saturation. This should be a good upper limit, as it is sufficient to saturate fluctuations when the initial amplitude of the noise fluctuations is $<10^{-5} \lambda$. In any event, the required number of e-foldings is very likely to be within a factor of two of this value.

For an infinitely steep initial velocity gradient $\left(H_{0}=0\right)$, one finds that the minimum wavelength for which the $\mathrm{KH}$ instability produces saturated fluctuations before its growth is stopped by the expansion of the boundary layer is $\lambda_{\mathrm{KH}} \sim$ $220 \delta / R$, which is below the inner viscous scale for values of $R$ large enough to observe a mixing transition. For a finite initial velocity gradient, one obtains the results shown in figure 10 . The figure shows curves above which $\mathrm{KH}$ of that normalized wavelength can meet our saturation criterion, overlaid on the normalized length versus the $R$ plane of figure 11. One concludes that, if $\mathrm{KH}$ at small-scale shear layers is required to initiate the population of the turbulent spectrum, then these shear layers must form in a way that keeps them initially quite abrupt.

\section{Conclusion}

We have seen that the development of turbulent flow in HEDP experiments, in the sense of having produced a mixing transition on a scale large enough to diagnose, is somewhat difficult to accomplish and has not been achieved to date. In this context, it is worth mentioning one result that was not discussed above. Hansen et al [52] published data and theory from an experiment in which a flowing HEDP plasma interacted with and stripped away mass from a denser cloud of material, finding clear evidence that in this case the mass stripping was turbulent. The turbulence, however, was on too small a scale to diagnose. The problem of observing turbulence and its onset in HEDP flows is one of several examples of mixing in unsteady flows that were presented at the 'Turbulent Mixing and Beyond' conference. 
In the above, after providing a discussion of the properties of HEDP hydrodynamic systems and the methods of doing such experiments, we discussed studies to date of the three primary instabilities: RM, RT and KH. The first two of these have been systematically observed, but have not yet produced a system with a clear transition to turbulence. The $\mathrm{KH}$ instability remains to be systematically observed in its pure form, although some related effects such as spike tip broadening have been seen. However, the KH effects seen in some simulations of RT systems and supersonic jets have not been seen to date in experiments.

We offered three observations that are speculative regarding the observations. We discussed the model of Zhou et al [35] regarding the onset of turbulence in time-dependent systems, and noted that their time-dependent condition is roughly equivalent to the Reynolds-number threshold of Dimotakis in that eddies will dissipate by turbulence in about one eddy-turnover time. We suggested that a plausible explanation of the absence of $\mathrm{KH}$ in several experimental systems may be that finite velocity gradients have quenched the instability. Finally, we argued that despite the smearing of the shear layer caused by viscous diffusion, $\mathrm{KH}$ instabilities have the potential to contribute to the generation of fluctuations at all scales so long as the local shear layers are formed with a sufficiently steep velocity gradient.

\section{Acknowledgments}

We acknowledge useful discussions with Harry F Robey, Ye Zhou, Dmitri D Ryutov and Bruce A Remington of the Lawrence Livermore National Laboratory. Harry Robey specifically first pointed out to us that in some of these systems the $\mathrm{KH}$ growth rate does not exceed the velocity gradient threshold. This research was sponsored by the National Nuclear Security Administration under the Stewardship Science Academic Alliances program through DOE Research grants DE-FG52-07NA28058, DE-FG52-04NA00064 and other grants and contracts.

\section{References}

[1] Davidson R C 2002 National, Research, Council, and Report, High-Energy-Density Physics: The X-Games of Contemporary Science (Washington DC: National Academies Press)

[2] Drake R P 2006 High Energy Density Physics: Fundamentals, Inertial Fusion and Experimental Astrophysics (Berlin: Springer)

[3] Tennekes H and Lumley J L 1972 A First Course in Turbulence (Cambridge, MA: MIT Press)

[4] Kolmogorov A N 1962 A refinement of previous hypotheses concerning the local structure of turbulence in a viscous incompressible fluid at high Reynolds number J. Fluid Mech. $\mathbf{1 3} 82$

[5] Zhang Y, Drake R P and Glimm J 2007 Numerical evaluation of impact of laser preheat on interface structure and stability Phys. Plasma 14062703

[6] Dimotakis P E 2000 The mixing transition in turbulent flows J. Fluid Mech. 40969

[7] Ryutov D D, Drake R P, Kane J, Liang E, Remington B A and Wood-Vasey M 1999 Similarity criteria for the laboratory simulation of supernova hydrodynamics Astrophys. $J$. 518821
[8] Robey H F 2004 The effects of viscosity and mass diffusion in hydrodynamically unstable plasma flows Phys. Plasmas 114123

[9] Zhou Y 2007 Unification and extension of the similarity scaling criteria and mixing transition for studying astrophysics using high energy density laboratory experiments or numerical simulations Phys. Plasma 14 082701

[10] Ryutov D D, Derzon M S and Matzen M K 2000 The physics of fast Z pinches Rev. Mod. Phys. 72167

[11] Kuranz C C, Blue B E, Drake R P, Robey H F, Hansen J F, Knauer J P, Grosskopf M J, Krauland C and Marion D C 2006 Dual, orthogonal, backlit pinhole radiography in Omega experiments Rev. Sci. Instrum. 77 10E327 1

[12] Aglitskiy Y, Lehecka T, Obenschain S, Pawley C, Brown C M and Seely J 1999 X-ray crystal imagers for inertial confinement fusion experiments [invited] Rev. Sci. Instrum. 70530

[13] Park H S et al 2006 High-energy K alpha radiography using high-intensity, short-pulse lasers Phys. Plasmas $\mathbf{1 3}$ 056309

[14] Velikovich A L 1996 Analytic theory of Richtmyer-Meshkov instability for the case of reflected rarefaction wave Phys. Fluids 81666

[15] Glendinning S G et al 2003 Effect of shock proximity on Richtmyer-Meshkov growth Phys. Plasmas 101931

[16] Kane J, Drake R P and Remington B A 1999 An evaluation of the Richtmyer-Meshkov instability in supernova remnant formation Astrophys. J. 511335

[17] Drake R P 2005 Hydrodynamic instabilities in astrophysics and in laboratory high-energy-density systems Plasma Phys. Control Fusion 417 B419

[18] Dimonte G and Schneider M 1996 Turbulent Rayleigh-Taylor instability experiments with variable acceleration Phys. Rev. E 543740

[19] Dimonte G et al 2004 A comparative study of the turbulent Rayleigh-Taylor instability using high-resolution three-dimensional numerical simulations: the Alpha-Group collaboration Phys. Fluids 161668

[20] Alon U, Shvarts D and Mukamel D 1993 Scale-invariant regime in Rayleigh-Taylor bubble-front dynamics Phys. Rev. E 481008

[21] Alon U, Hecht J, Ofer D and Shvarts D 1995 Power laws and similarity of Rayleigh-Taylor and Richtmyer-Meshkov mixing fronts at all density ratios Phys. Rev. Lett. 74534

[22] Waddell J T, Niederhaus C E and Jacobs J W 2001 Experimental study of Rayleigh-Taylor instability: Low Atwood number liquid systems with single-mode initial perturbations Phys. Fluids 131263

[23] Miles A R, Blue B, Edwards M J, Greenough J A, Hansen J F, Robey H F, Drake R P, Kuranz C and Leibrandt D R 2005 Transition to turbulence and effect of initial conditions on three-dimensional compressible mixing in planar blast-wave-driven systems Phys. Plasmas 120563171

[24] Kane J, Arnett D, Remington B A, Glendinning S G, Wallace R, Rubenchik A and Fryxell B A 1998 2nd Oak Ridge Symp. on Atomic and Nuclear Astrophysics (Oak Ridge, TN)

[25] Calder A et al 2002 On validating an astrophysical simulation code Astrophys. J. 143201

[26] Remington B A, Weber S V, Marinak M M, Haan S W, Kilkenny J D, Wallace R J and Dimonte G 1995 Single-mode and multimode Rayleigh-Taylor experiments on Nova Phys. Plasmas 2241

[27] Budil K S, Remington B A, Peyser T A, Mikaelian K O, Miller P L, Woolsey N C, Wood-Vasey W M and Rubenchik A M 1996 Experimental comparison of classical versus ablative Rayleigh-Taylor instability Phys. Rev. Lett. 764536

[28] Drake R P et al 2004 Nonlinear mixing behavior of the three-dimensional Rayleigh-Taylor instability at a decelerating interface Phys. Plasmas 112829 
[29] Miles A R, Braun D G, Edwards M J, Robey H F, Drake R P and Leibrandt D R 2004 Numerical simulation of supernova-relevant laser-driven hydro experiments on OMEGA Phys. Plasmas 113631

[30] Robey H F et al 2001 An experimental testbed for the study of hydrodynamic issues in supernovae Phys. Plasmas 82446

[31] Kane J, Arnett D, Remington B A, Glendinning S G, Castor J, Wallace R, Rubenchik A and Fryxell B A 1997 Supernova-relevant hydrodynamic instability experiments on the Nova laser Astrophys. J. 478 L75

[32] Kifonidis K, Plewa T, Janka H-T and Muller E 2003 Non-spherical core collapse supernovae. I. Neutrino-driven convection, Rayleigh-Taylor instabilities, and the formation and propagation of metal clumps Astron Astrophys. 408621

[33] Kifonidis K, Plewa T, Sheck L, Janka H-T and Muller E 2006 Non-spherical core collapse supernovae. II. The late-time evolution of globally anisotropic neutrino-driven explosions and their implications for 1987A Astron Astrophys. 457963

[34] Robey H F, Zhou Y, Buckingham A C, Keiter P, Remington B A and Drake R P 2003 The onset of turbulence in high Reynolds number, accelerated flows. Part II. Experiment Phys. Plasmas 10614

[35] Zhou Y et al 2003 Progress in understanding turbulent mixing induced by Rayleigh-Taylor and Richtmyer-Meshkov instabilities Phys. Plasmas 101883

[36] Caulfield C P and Peltier W R 2000 The anatomy of the mixing transition in homogeneous and stratified free shear layers J. Fluid Mech. $\mathbf{4 1 3} 1$

[37] Klaassen G P and Peltier W R 1985 The onset of turbulence in finite-amplitude Kelvin-Helmholtz billows J. Fluid Mech. 1551

[38] Chandrasekhar S 1961 Hydrodynamic and Hydromagnetic Stability (New York: Dover)

[39] Gerwin R A 1968 Stability of the interface between two fluids in relative motion Rev. Mod. Phys. 40652
[40] Foster J M et al 2005 High-energy-density, laboratory-astrophysics studies of jets and bow shocks Astrophys. J. Lett. $634 \mathrm{~L} 77$

[41] Dimotakis P E 2005 Turbulent Mixing Annu. Rev. Fluid Mech. 37329

[42] Blondin J M, Fryxell B A and Konigl A 1990 The structure and evolution of radiatively cooling jets Astrophys. $J$. 360370

[43] Xu J J, Hardee P E and Stone J M 2000 The stability of radiatively cooled jets in three dimensions Astrophys. $J$. 543161

[44] Gardiner T A, Frank A, Jones T W and Ryu D 2000 Influence of magnetic fields on pulsed, radiative jets Astrophys. $J$. 530834

[45] O'Sullivan S and Ray T P 2000 Numerical simulations of steady and pulsed non-adiabatic magnetised jets from young stars Astron. Astrophys. 363355

[46] Moraghan A, Smith M D and Rosen A 2006 Velocity study of axisymmetric protostellar jets with molecular cooling Mont. Not. R. Astron. Soc. 3711448

[47] Plewa T 1993 Fast radiative shocks in dense media. 2. Details of the dynamics Acta Astron. $\mathbf{4 3} 235$

[48] Rosen A and Smith M D 2004 Numerical simulations of highly collimated protostellar outflows - the effects of relative density Astron. Astrophys. 413593

[49] Hardee P E and Stone J M 1997 The stability of radiatively cooling jets. Linear analysis. Astrophys. J. 483121

[50] Hurricane O A 2008 Design for a high energy density Kelvin-Helmholtz experiment High Energy Density Phys. doi:10.1016/j.hedp.2008.02.002

[51] Esch R E 1957 The instability of a shear layer between two parallel streams J. Fluid Mech. 3289

[52] Hansen J F, Robey H F, Klein R I and Miles A R 2007 Experiment on the mass stripping of an interstellar cloud following shock passage Astrophys. J. 662379 